\title{
Effects of Immediate or Delayed Estradiol on Behavior in Old Menopausal Macaques on Obesogenic Diet
}

\author{
Kristine Coleman, ${ }^{1,2}$ Nicola D. Robertson, ${ }^{2}$ Adriane Maier, ${ }^{2}$ and Cynthia L. Bethea $\mathbb{D D}^{1,3,4}$ \\ ${ }^{1}$ Division of Neuroscience, Oregon National Primate Research Center, Beaverton, OR 97006, USA \\ ${ }^{2}$ Division of Comparative Medicine, Behavioral Sciences Unit, Oregon National Primate Research Center, Beaverton, \\ OR 97006, USA \\ ${ }^{3}$ Division of Reproductive Sciences, Oregon National Primate Research Center, Beaverton, OR 97006, USA \\ ${ }^{4}$ Department of Obstetrics and Gynecology, Oregon Health and Science University, Portland, OR 97201, USA
}

Correspondence should be addressed to Cynthia L. Bethea; betheac@ohsu.edu

Received 11 May 2018; Revised 12 July 2018; Accepted 22 July 2018; Published 27 September 2018

Academic Editor: Gordon Fisher

Copyright (c) 2018 Kristine Coleman et al. This is an open access article distributed under the Creative Commons Attribution License, which permits unrestricted use, distribution, and reproduction in any medium, provided the original work is properly cited.

\begin{abstract}
Macaques have served as effective models of human disease, including pathological processes associated with obesity and the metabolic syndrome. This study approached several questions: (1) does a western-style diet (WSD) contribute to sedentary behavior or is sedentary behavior a consequence of obesity and (2) does estradiol (E) hormone therapy offset WSD or ameliorate sedentary behavior? We further questioned whether the timing of E administration (immediately following hysterectomy, ImE; or after a 2-year delay, DE) would impact behavior. Focal observations were taken on the animals in social housing over a period of 2.5 years before and after initiation of the WSD and hysterectomy. In addition, anxiety was assessed through the Human Intruder and Novel Object Tests. All animals gained weight, but ImE delayed the time to maximum weight achieved at 18 months. Over the course of the study, ImE-treated monkeys spent more time "alone" and less time in "close social" contact than placebo-controls. The DE-treated monkeys were not different from placebo-controls in these 2 outcomes. The placebo-control group exhibited more "self-groom" behavior, an indicator of anxiety, than did the ImE-treated group, and DE-treated animals approached levels observed in the ImE-treated animals. All animals exhibited an increase in "consume" behavior over time with no statistical difference between the groups. By the end of the protocol, the placebo-control group exhibited less activity compared to ImE + DE-treated animals combined. Animals also showed increased anxiety after starting on the WSD in the Human Intruder Test and the Novel Object Test. In summary, the data indicated that WSD per se promoted increased consummatory behavior, sedentary behavior, and anxiety-type behaviors, whereas ImE promoted activity. Thus, WSD may precipitate the behaviors observed in humans who then become obese, sedentary, anxious, and socially isolated. ImE replacement ameliorates some of these behaviors, but not all.
\end{abstract}

\section{Introduction}

Middle-aged women today face two major health issues, obesity and menopause, both of which precipitate a number of health problems and a decline in the quality-of-life indices. The symptoms of menopause and long-term physiological deterioration may be ameliorated by proper hormone therapy. However, due to the overgeneralization of results from the Women's Health Initiative (WHI), many women and their physicians are still concerned about its use.
The WHI administered conjugated equine estrogens to women who were on average 10-12 years past menopause $[1,2]$. Nonetheless, the use of estradiol-17 $\beta$ (E) administration during the perimenopause period has yielded positive outcomes with respect to mood [3, 4], cognition [5], carotid intima-media thickness (CIMT; [6]), metabolism and body composition [7], lung function [8], and immune function [9] (including multiple sclerosis [10]), compared with no treatment or delayed treatment. Still, the outcomes of clinical trials with $\mathrm{E}$ (and $\mathrm{E}$ conjugates) vary from trial to 
trial, whereas the effects of $\mathrm{E}$ replacement in laboratory animal studies have been robust and repeatable. A major factor in the difference between the human and animal studies is diet. Unlike the typical American diet, laboratory animal chow is low in fat and refined sugar, but high in micronutrients.

A large portion of the US population eats a "westernstyle diet" (WSD) that is high in fat and refined sugar. Twothirds of the US population is overweight or obese [11], and 114 million people exhibit symptoms of pre diabetes or frank diabetes (American Diabetes Association). Weight gain and changes in body morphology often accompany menopause [12]. Unfortunately, today many women enter menopause already obese. As obese women enter menopause, an obvious speculation is that their risk for disease could increase over the presence of either risk factor alone [13].

Obesity plays a role in metabolic disease, which exhibits a constellation of symptoms including large waist circumference or elevated waist-to-hip ratio, elevated fasting serum glucose and serum triglycerides, abnormal cholesterol, insulin resistance, hypertension, and depression. Metabolic disease is clearly linked to type 2 diabetes and cardiovascular disease. Low activity levels and a sedentary lifestyle contribute significantly to the risk of developing metabolic syndrome. Notably, menopause is one of the greatest risk factors for developing metabolic disease. $\mathrm{E}$ is also anorexic, and its loss at menopause promotes weight gain.

Although lifestyle modification is the preferred treatment for metabolic disease, well-established coping behaviors, such as overeating and a sedentary lifestyle, are extremely difficult to change in humans. Similarly, in macaques, lack of exercise and stress, such as that caused by lack of conspecific contact, can also contribute to weight gain. Moreover, age and female sex also increase weight if housing is the same [14].

Based upon the above information, we wondered whether hormone therapy would maintain its beneficial effects in women if they were eating WSD and/or metabolically compromised. Nonhuman primates have provided important information related to human reproduction and cardiovascular physiology. For example, in surgically menopausal cynomolgus macaques on a high fat-high sugar diet, $\mathrm{E}$ administration prevented the development of atherosclerosis observed in placebo-controls [15]. To further explore this question in multiple systems, we established a colony of old surgically menopausal (ovohysterectomized, $\mathrm{OvH}$ ) WSD-fed rhesus macaques. In addition, we sought to model single hormone therapy administered during the perimenopause with $\mathrm{E}$ replacement immediately upon $\mathrm{OvH}$ ( $\operatorname{ImE}$ ) versus $\mathrm{E}$ administered long after menopause (as in the WHI) with E replacement 2 years after OvH (DE; 2 monkey years $=6-8$ human years). We have reported that WSD blunted or abolished the positive effects of immediate $\mathrm{E}$ replacement (ImE) on gene expression in the serotonin neural system [16], in circadian activity [17], and in a large number of metabolic parameters [18-20]. All of the monkeys gained weight, although $\operatorname{ImE}$ delayed the increase; by the end of the study, all monkeys reached the same average higher weight. The same pattern was observed in the percent of truncal fat, and in the area under the insulin and glucose curves generated from IVGTT at 6-month intervals. Rhesus macaques appear resistant to atherosclerosis [21, 22], unless the diet contains high cholesterol and $40 \%$ of calories from peanut oil [23]. Likewise, atherosclerosis was absent in coronary arteries of our WSD-fed rhesus macaques, but preliminary data suggest that WSD caused an expansion between elastin lamellae in coronary arteries (carotid intima-media thickness, CIMT), and E administration had no effect. CIMT leads to stiffness and elevated blood pressure. Finally, the group with $\mathrm{E}$ administration after 2 years on WSD (DE) was similar to the placebo group in most metabolic outcomes.

To determine if old, surgically menopausal, WSD-fed rhesus macaques modeled human behavior, as well as show differences in behavior in the presence or absence of $\mathrm{E}$ replacement, we performed longitudinal focal observations and longitudinal provoked anxiety tests on all of the animals. We hypothesized that $\operatorname{ImE}$ would promote increased activity and that the social housing would enable more exercise. Herein, we report the results of the behavioral observations. In addition, we comment on the process of socially housing old female rhesus macaques that were previously housed in different conditions.

\section{Materials and Methods}

The ONPRC animal care program is compliant with the laws and regulations of the United States Animal Welfare Act and is accredited by AAALAC-International. The ONPRC Institutional Animal Care and Use Committee approved this study.

2.1. Subjects. Twenty-eight aged (at least 17 years of age) female rhesus macaques (Macaca mulatta) were potential subjects for this study. The study was intended to run for 3 years, at which time the majority of monkeys would be 20 years old. Age-related pathologies manifested, and so the study was terminated after 2.5 years. Monkeys were fed standard chow (Lab Diet, Inc., St. Louis, MO) until the start of this study. After 3 months of baseline monitoring, they were switched to a "western-style diet" (WSD) also called a "typical American diet" (TAD; Lab Diet) twice daily. Regular monkey chow provides calories with 13\% fat, $69 \%$ complex carbohydrates (includes 6\% sugars), and 18\% protein. In contrast, WSD provided calories with $36 \%$ fat, $44 \%$ carbohydrates (includes $18.5 \%$ sugars), and $18 \%$ protein. Monkeys were given fresh produce each day, and water was provided freely through automatic lixit systems. The lights were on $12 \mathrm{hr}$ per day. Subjects were given enrichment such as toys, foraging devices, radio, and television to ensure their psychological health and well-being.

The monkeys were socially housed in indoor pens (approximately $3.7 \mathrm{~m} \times 2.1 \mathrm{~m} \times 2.1 \mathrm{~m}$ ) situated in rooms containing up to 32 cage-housed monkeys. Each pen was located in a different room. Our goal was to place 3-4 animals in each pen; however, social introduction attempts were not always successful, and sometimes resulted in 
aggression. ONPRC behavioral and husbandry staff closely monitored the pens, and animals that were overly aggressive were removed from the groups. All animals were socially housed throughout the study. As shown in Table 1, the pens finally contained 2,3 , or 4 animals $(n=24)$ with ongoing attrition.

Subjects were maintained on normal monkey chow during acclimation to the groups. After acclimation, subjects were fed WSD for approximately 6 weeks and then ovohysterectomized $(\mathrm{OvH}$; spayed). $\mathrm{OvH}$ was performed because the old female macaques were to receive $\mathrm{E}$ for at least 30 months ( 2.5 years), and endometrial hyperplasia would be expected. Since older macaques are also more susceptible to cancer, $\mathrm{OvH}$ was used to decrease risk during the treatment period.

All animals were weighed at baseline 0 (before start of WSD), baseline 1 (before $\mathrm{OvH}$ ), and every 3 months during the first year and every 6 months thereafter. Weight was also obtained prior to surgical procedures.

The animals were trained to run into a tunnel of smaller cages upon a hand signal. Once in the small cages, the animals could be separated for medications, injections, venipuncture, or examination.

2.2. Surgery. Subjects were ovohysterectomized $(\mathrm{OvH})$ via the laparotomy approach by ONPRC surgical personnel. For surgeries, each animal was removed from the group, sedated with ketamine $(10 \mathrm{mg} / \mathrm{kg})$, and transported to a surgical suite. After the animals had fully recovered, they were returned to their social group.

2.3. Treatments. The old WSD-fed macaques received (1) placebo for 30 months, or (2) E immediately upon hysterectomy for 30 months (ImE), or (3) placebo for 24 months and delayed $\mathrm{E}$ for an additional 6 months (2.0 years interval; DE for 6 months). The protocol was started with 8 animals in each treatment group and 4 extra nontreated animals. The animals were organized into mixed cohorts of 8 , each of which consisted of 2-3 different pens. Each pen contained animals with different treatments and different ranks. The treatment and assessment protocols were staggered 1 month by cohort to obtain all measurements on all animals in a technically feasible manner. In the first 6 months of the study, 4 animals were deemed unsuitable. They were replaced, and protocols were further staggered for the replacements. Afterwards, attrition occurred due to age-related diseases.

Estrogen was administered via Silastic capsules implanted subcutaneously in the periscapular region. One Silastic capsule (3.5 to $4.5 \mathrm{~cm}$ depending on metabolism; inner diameter, 0.132 in., outer diameter, 0.183 in.; Dow Corning, Mid-land, MI) was packed with crystalline estradiol (1,3,5_10-estratrien-3,17-diol, Steraloids, Wilton, NH). The implants were intended to achieve $\mathrm{E}$ concentrations between 70 and $100 \mathrm{pg} / \mathrm{ml}$ in the serum. An empty Silastic capsule constituted the placebo treatment.

Monkey metabolism can be highly variable, so serum E was measured every 2 months in the old WSD-fed macaques starting shortly after hysterectomy. At any time during the
TABLE 1: The group, treatment, and ranks of the animals at the start and end of the study.

\begin{tabular}{|c|c|c|c|c|}
\hline Animal & Pen & Treatment & $\begin{array}{l}\text { Rank } \\
\text { Start }\end{array}$ & $\begin{array}{c}\text { Rank } \\
\text { End }\end{array}$ \\
\hline$\overline{\mathrm{A} 1}$ & 1 & Placebo & $\mathrm{D}$ & $\mathrm{D}$ \\
\hline A2 & 1 & $\operatorname{ImE}$ & $S$ & $\sim$ \\
\hline A3 & 1 & Placebo & $\mathrm{M}$ & S \\
\hline A4 & 1 & Placebo & $\mathrm{M}$ & $\mathrm{M}$ \\
\hline B1 & 2 & $\operatorname{ImE}$ & $\mathrm{D}$ & $\mathrm{D}$ \\
\hline B2 & 2 & $\mathrm{DE}$ & $\mathrm{M}$ & $\mathrm{M}$ \\
\hline B3 & 2 & $\mathrm{DE}$ & $\mathrm{S}$ & S \\
\hline B4 & 2 & Placebo & $\mathrm{M}$ & $\sim$ \\
\hline $\mathrm{C} 1$ & 3 & $\mathrm{DE}$ & $\mathrm{D}$ & $\mathrm{D}$ \\
\hline $\mathrm{C} 2$ & 3 & Placebo & $\mathrm{M}$ & $\mathrm{M}$ \\
\hline $\mathrm{C} 3$ & 3 & $\operatorname{ImE}$ & $S$ & $\mathrm{~S}$ \\
\hline $\mathrm{C} 4$ & 3 & $\operatorname{ImE}$ & $\mathrm{M}$ & $\sim$ \\
\hline D1 & 4 & $\mathrm{DE}$ & $\mathrm{M}$ & $S$ \\
\hline D2 & 4 & $\mathrm{DE}$ & $S$ & $\mathrm{D}$ \\
\hline D3 & 4 & Placebo & $\mathrm{D}$ & $\sim$ \\
\hline D4 & 4 & $\operatorname{ImE}$ & M & $\sim$ \\
\hline E1 & 5 & $\operatorname{ImE}$ & $\mathrm{M}$ & $\mathrm{M}$ \\
\hline E2 & 5 & $\mathrm{DE}$ & $\mathrm{D}$ & $\mathrm{D}$ \\
\hline E3 & 5 & Placebo & $S$ & $S$ \\
\hline $\mathrm{F} 1$ & 6 & $\mathrm{DE}$ & M & $\sim$ \\
\hline F2 & 6 & $\operatorname{ImE}$ & $S$ & $\mathrm{D}$ \\
\hline F3 & 6 & $\mathrm{DE}$ & $\mathrm{D}$ & $\mathrm{S}$ \\
\hline F4 & 6 & Placebo & M & $\sim$ \\
\hline G1 & 7 & Placebo & $\mathrm{D}$ & $\sim$ \\
\hline G2 & 7 & Placebo & $S$ & $S$ \\
\hline G3 & 7 & $\operatorname{ImE}$ & M & $\mathrm{D}$ \\
\hline $\mathrm{H} 1$ & 8 & $\operatorname{ImE}$ & S & S \\
\hline $\mathrm{H} 2$ & 8 & $\operatorname{ImE}$ & $\mathrm{D}$ & $\mathrm{D}$ \\
\hline
\end{tabular}

$\sim$ denotes missing data, which by 30 months was largely due to attrition. $\mathrm{D}=$ dominant (highest ranking) monkey. $\mathrm{M}=$ midranking monkey(s). $\mathrm{S}=$ subordinate (lowest ranking) monkey. Please note that the last 4 animals (G 2-3, H1-2) were initially extras, but they were added after other animals were removed from the study.

duration of the protocol, if serum E concentrations were higher than $120 \mathrm{pg} / \mathrm{ml}$ in an individual measurement, the capsule was replaced with a smaller capsule, which was used for that individual henceforth. When serum $E$ levels declined below $50 \mathrm{pg} / \mathrm{ml}$, the implants were replaced. There is a surge of $\mathrm{E}$ immediately after implantation that gradually declines and stabilizes; so, monitoring every 2 months enabled us to maintain the goal on average [16].

2.4. Behavioral Assessments. We used two methods to assess behavior and anxiety in this study: home environment assessments and provoked response tests (Human Intruder Test (HIT) and Novel Object Test (NOT)). This combined testing paradigm provided a more comprehensive picture of the emotional states of the animals than a solitary assessment method. Home environment assessments examine response to everyday, naturalistic events such as interactions with conspecifics and care staff [24]. Provoked response tests assess unconditioned response to various threatening or potentially threatening stimuli. For all tests, the observer was blind to the experimental treatment of the animals. The Observer software (Noldus Information Technology, 
Wageningen, Netherlands) was used to score behavior (both live and from video). The home environment assessments were performed at 5 time points, and the provoked response tests were performed at 4 time points during the study (Table 2).

The behavioral observations were obtained as part of a larger study involving these animals ([16]).

\subsubsection{Home Cage Behavioral Assessments. A highly trained} and experienced observer took 10-min continuous focal observations [25] on the monkeys 2-3 times/week for 2-3 weeks (for a total of 60 min of observations per individual) at each time point. The observer, with whom the monkeys were familiar, entered the room and stood next to the pen for $10 \mathrm{~min}$ to allow the animals to acclimate to her presence. Monkeys are used to having people in their rooms and typically ignored her after a few minutes. She then began to record the behavior of the focal individual, one at a time, directly onto a laptop computer for $10 \mathrm{~min}$ each. To limit time of day effects, observations were taken between 12: 00-3:00 pm. The order of observations was randomized, to ensure that animals had an equal probability of being observed in relationship to daily events such as afternoon feedings. Table 3 details the ethogram of the behaviors coded in this study. Behaviors were organized into three behavioral classes: social behavior, nonsocial behavior, and events. Behaviors within the social and nonsocial behavioral class were mutually exclusive, but could co-occur with behaviors from the other class (e.g., an individual cannot be alone and touching another animal, but could be alone and stationary). Behaviors that naturally occurred in relatively short durations, such as scratches or threats, were classified as "events." Behavioral variables were calculated as percent of time the animal engaged in that behavior (social and nonsocial behavior) or the frequency with which the behavior occurred (events).

2.4.2. Human Intruder Test (HIT). This test was designed to measure behavioral inhibition and anxiety in rhesus monkeys [26]. It assesses the behavioral response of a monkey to three stressful conditions: being alone in an unfamiliar cage, being in the presence of a human stranger whose gaze is diverted (Profile; a nonthreatening social stimulus), and being in the presence of a human stranger making direct eye contact (Stare; a threatening social stimulus).

The monkeys were temporarily removed from their social group, one at a time, brought to a dedicated behavioral testing room, placed alone in a standard monkey cage in a novel room, and videotaped from behind a one way mirror. Every animal in the group was tested on the same day, in a randomized order. The test began with a 12-minute acclimation period (alone 1). After this time period, an unfamiliar human entered the testing room and approached to $0.3 \mathrm{~m}$ of the cage, taking care not to make eye contact with the monkey. The human presented her facial profile to the monkey for 2 minutes (Profile). The human then left the room, leaving the monkey alone for another 2 minutes (alone 2). The human stranger re-entered the room, approached to $0.3 \mathrm{~m}$ of the cage, and made continuous, direct eye contact for 2 minutes (Stare). Direct eye contact is generally considered to be a threatening facial expression for monkeys. After the human intruder left, the monkey was videotaped for another $2 \mathrm{~min}$ (alone 3). Behaviors that were scored during this test include vocalizations, movement, and reaction to stranger, including freezing, fearful, and threatening expressions (Table 4). Behavioral variables were calculated as percent of time the animal engaged in that behavior.

2.4.3. Novel Object Test. This test was designed to test the monkey's reaction to various novel objects, including an ecologically relevant novel object with reward value (i.e., a piece of unfamiliar fruit), a brightly colored toy and a rubber snake. After the alone 3 period of the HIT, the intruder reentered the room and put various novel objects in the cage, each for $5 \mathrm{~min}$. Except for the novel food, all items were removed before new objects were introduced. The novel objects were in the following order: a novel food item such as kiwi, a brightly colored bird toy, Mr. Potato Head, and a rubber snake with a piece of apple (highly desirable item) on top. Mr. Potato Head was chosen as a potentially threatening stimulus because of the large eyes.

At the end of this test, the monkey was returned to her social group. Behaviors coded from this test included the latency to inspect (approached within $3 \mathrm{~cm}$ ), touch (intentional contact), and manipulate each item. We calculated a "Bold Score" based on an individual's reaction to the objects [27]. The scores went from 0 (low boldness; the monkey did not inspect any object within 10 seconds) to 12 (high boldness; the monkey inspected each of the items within 10 seconds).

Monkeys received their standard morning meal approximately two hours prior to the advent of the testing, to avoid the confound of hunger during the tests. Altogether, the HIT and NO tests took approximately 45 minutes.

2.5. Statistical Analysis. Not every animal underwent every procedure. Nine animals became ill and had to be removed from the study before 30 months; thus, there were a total of 20 animals that went through each behavioral time point (placebo $n=6 ; \operatorname{ImE}=7 ; \mathrm{DE}=7$ ).

For each test (home assessments, HIT, and NOT), we compared behavior across time and between treatment groups using a 2-way ANOVA followed by Sidak's multiple comparison post hoc test. One-way ANOVA or a $t$-test was used on data at the 30-month time point. Alpha values were set at 0.05 . Prism v7.0 software from GraphPad was used for all analyses. Eighteen home cage behaviors were monitored, and the number of behaviors that exhibited significant differences was relatively small. Therefore, adjustment for false discovery rate (FDR) was not necessary.

\section{Results}

Eighteen individual behaviors were scored during focal observations in the home pen environment (Table 3). Table 4 outlines the behaviors recorded in the HIT. Of these 
TABLE 2: Time points for behavioral testing (numbers in parentheses are mean $\pm S D$ ).

\begin{tabular}{|c|c|c|}
\hline Time point & Description & Anxiety test performed \\
\hline Baseline $0(\mathrm{~B} 0)$ & $\begin{array}{c}\text { Approximately } 2(1.9 \pm 0.7) \text { months after } \\
\text { introduction to the group; prior to introduction of } \\
\text { WSD and ovariectomy }\end{array}$ & $\begin{array}{c}\text { HIT } \\
\text { NOT } \\
\text { Focal observation }\end{array}$ \\
\hline Baseline 1 (B1) & $\begin{array}{c}\text { Approximately } 3(2.8 \pm 0.6) \text { months after } \mathrm{B} 0 \text {; animals } \\
\text { on high-fat diet; prior to ovohysterectomy }\end{array}$ & Focal observations \\
\hline Year 1 & Approximately $7.5(7.5 \pm 0.8)$ months after B1 & $\begin{array}{c}\text { HIT } \\
\text { NOT } \\
\text { Focal observations } \\
\text { HIT }\end{array}$ \\
\hline Year 2 & 12 months after year 1 & $\begin{array}{l}\text { NOT } \\
\text { Focal observations } \\
\text { HIT }\end{array}$ \\
\hline Year 2.5 & Prior to end of study & $\begin{array}{c}\text { NOT } \\
\text { Focal observations }\end{array}$ \\
\hline
\end{tabular}

TABLE 3: Ethogram of behaviors coded in the home cage assessments.

\begin{tabular}{|c|c|c|}
\hline Behavioral Class & Behavior & Definition \\
\hline \multirow{6}{*}{ Social behaviors (measured in percent of time) } & Groom & $\begin{array}{l}\text { Focal individual is picking at hair and/or skin of } \\
\text { another individual (focal can initiate or receive } \\
\text { behavior) }\end{array}$ \\
\hline & Proximity & $\begin{array}{c}\text { Focal individual is within arms length of another } \\
\text { individual without touching }\end{array}$ \\
\hline & Touch & Focal is in physical contact with another individual \\
\hline & Ventral contact & $\begin{array}{c}\text { Special case of touch, in which ventral surface of both } \\
\text { animals are in contact }\end{array}$ \\
\hline & Positive social behavior & $\begin{array}{c}\text { Combined behavior which includes groom, touch, or } \\
\text { ventral contact }\end{array}$ \\
\hline & Alone & $\begin{array}{c}\text { Focal individual is not in any social contact with } \\
\text { other monkeys }\end{array}$ \\
\hline \multirow{7}{*}{ Nonsocial behaviors (measured in percent of time) } & Stereotypical behavior & $\begin{array}{c}\text { Repetitive behavior with no apparent purpose, such } \\
\text { as pacing, circling, or poking eye }\end{array}$ \\
\hline & Consume & Handling and ingesting food and/or water \\
\hline & Locomote & Movement (e.g., walk, run) \\
\hline & Object play & $\begin{array}{l}\text { Focal individual manipulates object (e.g., toys or } \\
\text { structures in the corral) other than food }\end{array}$ \\
\hline & Self-groom & Focal individual grooms self \\
\hline & Sleep & $\begin{array}{c}\text { Focal individual is sitting with eyes closed, usually } \\
\text { huddled with other individuals }\end{array}$ \\
\hline & Stationary & $\begin{array}{c}\text { Focal individual sitting quietly, not engaged in other } \\
\text { behavior }\end{array}$ \\
\hline \multirow{7}{*}{ Events (measured as frequency) } & Aggression & Bite, hit, slap \\
\hline & Fear grimace & Focal individual bars teeth \\
\hline & Lipsmack & Rapid movement of lips \\
\hline & Scratch & Common usage \\
\hline & Threat & $\begin{array}{c}\text { Open mouth threat gesture (focal can initiate or } \\
\text { receive behavior) }\end{array}$ \\
\hline & Yawn & Common usage \\
\hline & Dominance-related behavior & $\begin{array}{c}\text { Combined behavior which includes aggression, } \\
\text { chase, displace, and threat }\end{array}$ \\
\hline
\end{tabular}

behaviors, only a few were either significantly altered or warrant mention in contrast to other behaviors.

As illustrated in Figure 1, animals treated with "immediate estradiol" (ImE) at the end of the OvH surgery spent more time "alone" (not in social contact with pen mates) during focal observations as the protocol progressed (treatment, 2-way ANOVA: $F(1,72)=6.622, p=0.012$ ). The
ImE-treated animals also showed a concomitant decrease in "close social" behavior as time "alone" increased during the study period. There was no apparent effect of DE. A combined group of placebo + DE-treated animals spent significantly more time in "close social" behavior than the ImEtreated group by the 30 -month time point $(t$-test $t(13)=$ 2.694; $p=0.018$ ). 
TABLE 4: Ethogram of behaviors coded during the Human Intruder Test.

\begin{tabular}{lc}
\hline Behavior & Operational definition \\
\hline Vigilant & $\begin{array}{c}\text { Subject's gaze is not directed toward human intruder } \\
\text { Tense body posture with no movement and no } \\
\text { vocalization }\end{array}$ \\
Locomotion & $\begin{array}{c}\text { Active behavior resulting in movement from original } \\
\text { location (e.g., moving across cage) }\end{array}$ \\
$\begin{array}{l}\text { Stationary } \\
\text { Stereotypical behavior }\end{array}$ & $\begin{array}{c}\text { Focal individual sitting quietly, not engaged in other } \\
\text { behavior }\end{array}$ \\
$\begin{array}{l}\text { Vocalizations } \\
\text { Yawn }\end{array}$ & $\begin{array}{c}\text { Repetitive behavior with no apparent purpose } \\
\text { Includes coo and shriek }\end{array}$ \\
Self-directed anxiety behavior & $\begin{array}{c}\text { Subject opens mouth very wide, baring upper teeth } \\
\text { Subject scratches or shakes (quick action of rotating } \\
\text { head and top of shoulders back and forth) }\end{array}$ \\
\hline
\end{tabular}

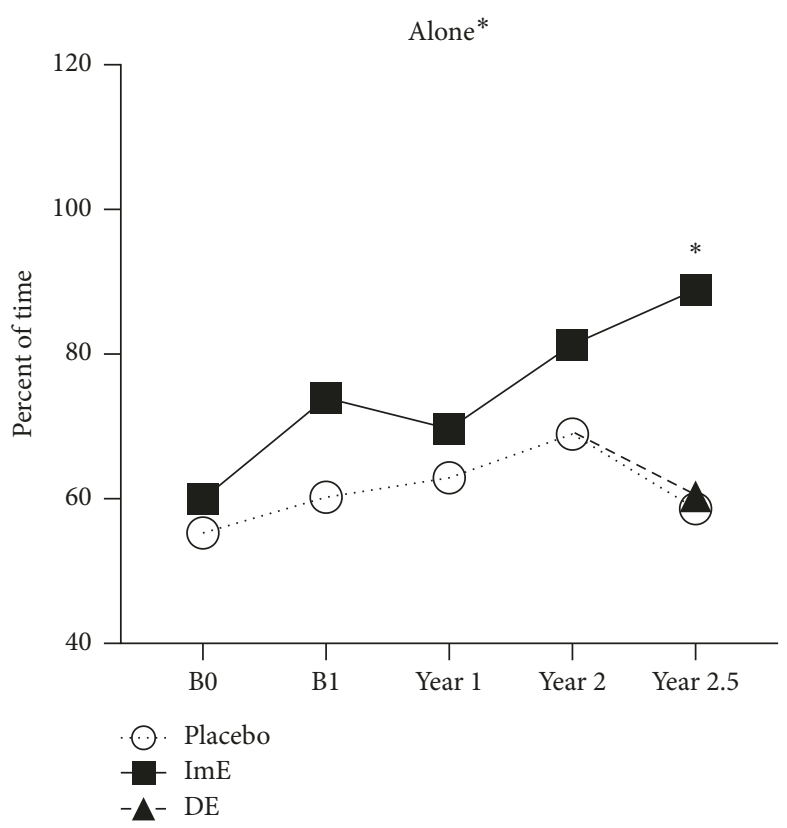

(a)

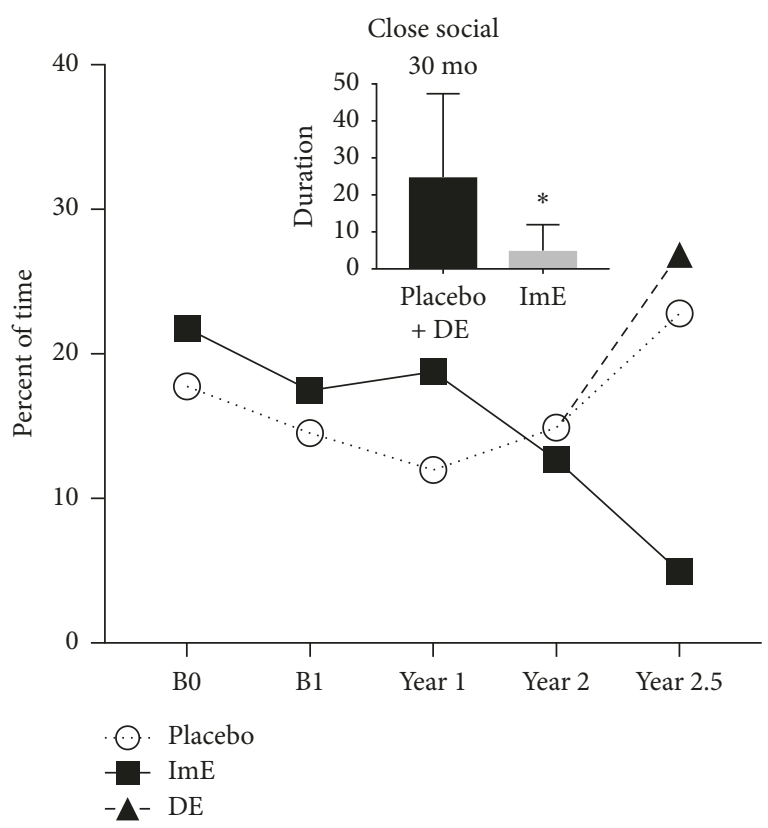

(b)

FIGURE 1: (a) The ImE-treated group exhibited higher duration of "alone" time during the protocol. In the 30-month (2.5 years) observations, there was a post hoc difference between the ImE group and the placebo or DE group (Bonferroni $p<0.05$ ). (b) The ImE-treated group showed a decline in "close social" behavior over time that was statistically manifested at the 30-month (2.5 years) time point (Inset). "Close social" behavior was significantly less in the ImE group when compared with the combined placebo + DE groups $(t$ test $p<0.017)$. (Title* different by 2-way ANOVA $(p<0.05)$; graph* different by post hoc test $(p<0.05))$.

Figure 2 shows that "consume" behavior (i.e., ingesting food and/or water) increased over time in both placebo- and ImE-treated groups (2-way ANOVA for time: $F(1,72)=$ 2.479; $p=0.05)$. DE appeared to decrease "consume" behavior at 30 months, but the variance and attrition of the old animals by this time point precluded statistical significance. Concomitantly, "locomote behavior," including large motions that would be detected by activity collars [17], exhibited a decreasing trend over time $(F(4,75)=2.257$; $p=0.071)$. At 30 months, "locomote" in the $\operatorname{ImE}+\mathrm{DE}$ combined group was higher than that in the placebo group $(t$ $(15)=2.382 ; p=0.033$ ).

"Self-groom" behavior was different between placeboand ImE-treated animals, but the groups had different baselines prior to any treatment (B0). Therefore, "selfgroom" was individually normalized (divided) by B0, and the ratios were analyzed with 2-way ANOVA (Figure 3). "Self-groom" was higher in the placebo-treated animals than in the ImE-treated animals $(F(1,51)=4.185 ; p=0.046)$. After 6 months of DE, "self-groom" behavior approached the average observed in the ImE group, suggesting that DE also decreased "self-groom" behavior.

There was no difference between the groups in stereotypical behavior (Figure 3), as determined with 2-way ANOVA $(F(1,78)=1.995 ; p=0.162)$.

Figure 4 illustrates results from the provoked anxiety tests. The animals had different personalities and thus different baseline values to start (B0), so they were individually 


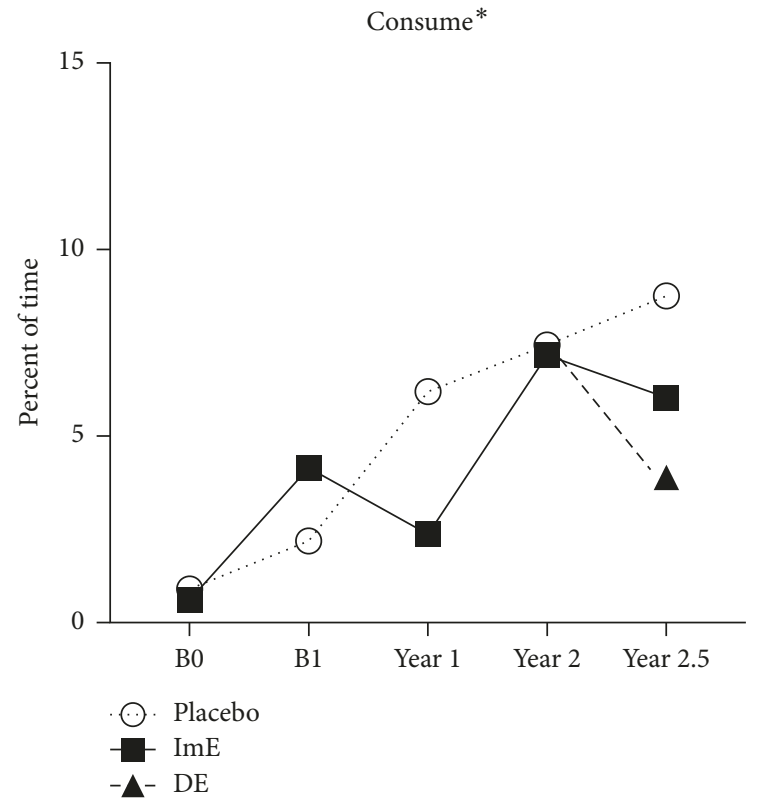

(a)

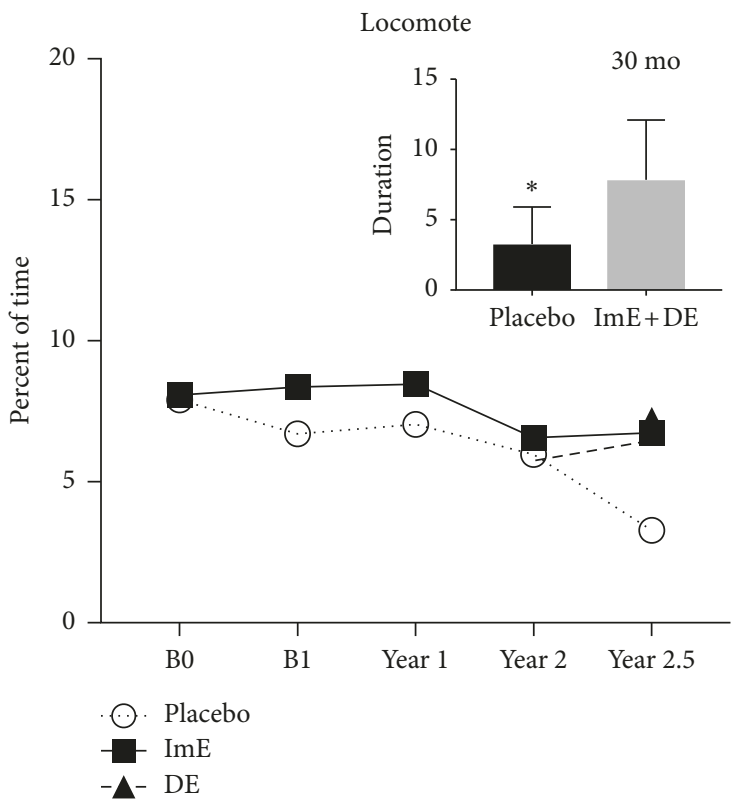

(b)

Figure 2: (a) There was an increase in "consume" behavior over time in all WSD-fed animals, but treatment with E had no significant effect. Comparison of the placebo group versus a combined group of $\operatorname{ImE}+\mathrm{DE}$ animals was not different by $t$-test. (b) Average "locomote" behavior was significantly higher in the combined ImE + DE animals compared to the placebo-control animals at the 30-month (2.5 years) time point $(t$-test, $p<0.033)$. (Title* different by 2 -way ANOVA $(p<0.05)$; graph* different by post hoc test $(p<0.05)$ ).

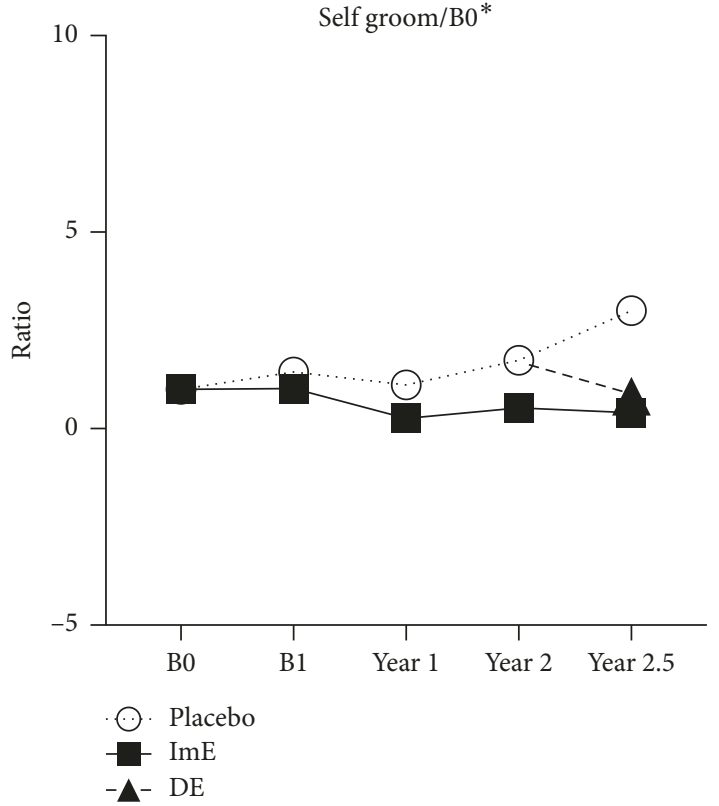

(a)

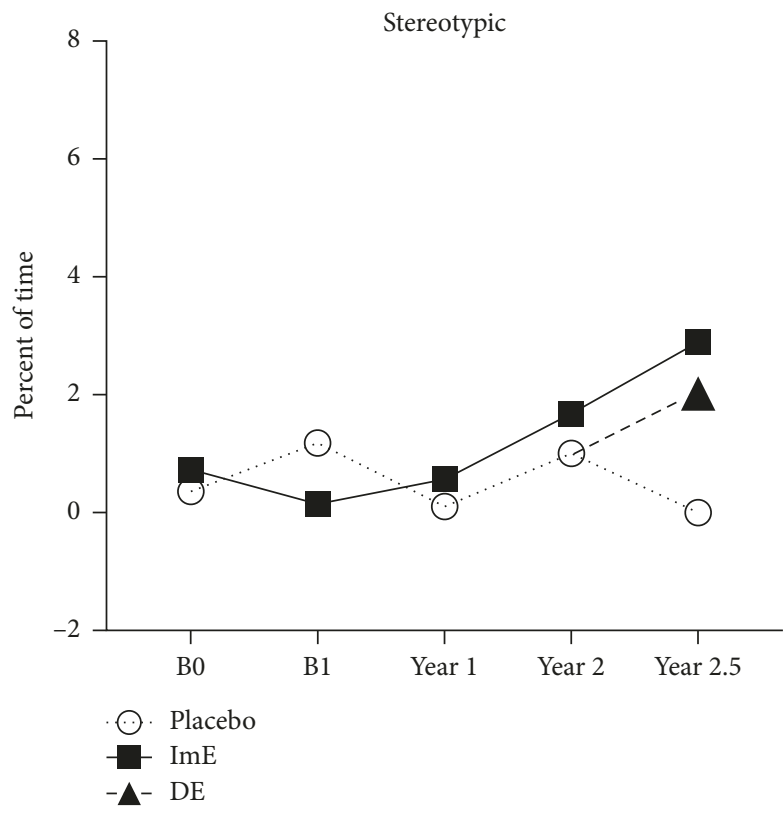

(b)

Figure 3: (a) Self-groom was significantly higher in the placebo animals than in the ImE treated animals $(p<0.045)$, but this was mostly due to the final time point. However, there was no difference over time. Of note, the placebo-controls showed a marked decrease in selfgrooming after DE was initiated. (b) There was no difference in "Stereotypical" behavior although the DE group approached the ImE group after 6 months of treatment. (Title* different by 2 -way ANOVA $(p<0.05)$; graph* different by post hoc test $(p<0.05)$ ).

normalized (divided) by their own B0. In the HIT, locomoteacclimate (loco acclim) in panel A reflects movement during the initial acclimation period (alone 1). "loco acclim" was not different with treatment. However, 2-way ANOVA indicated that a significant difference occurred across time $(F(3,62)=4.371 ; p=0.007)$. The "freeze profile" in panel B 


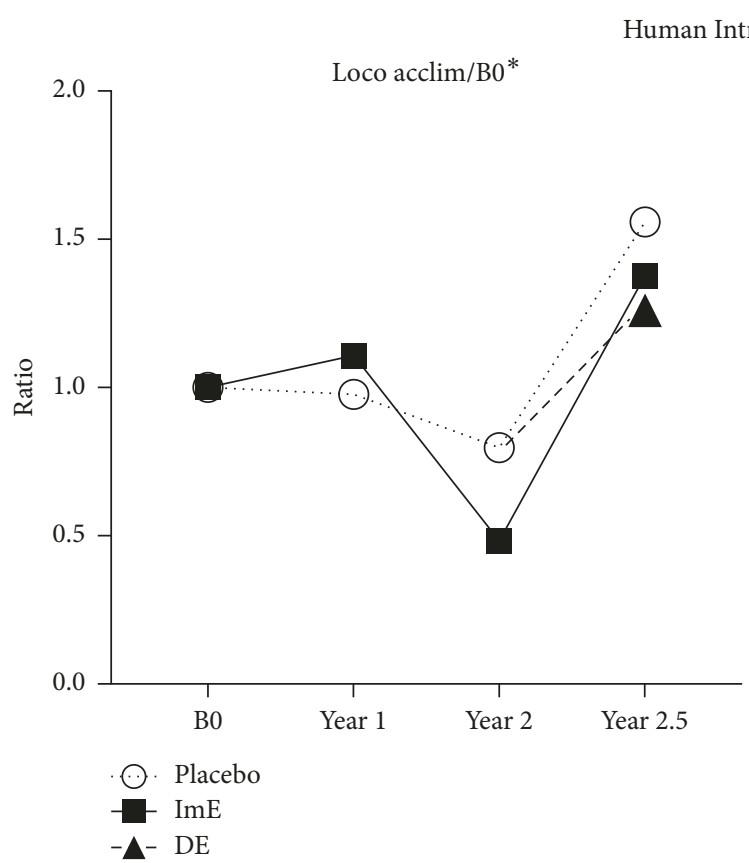

(a)

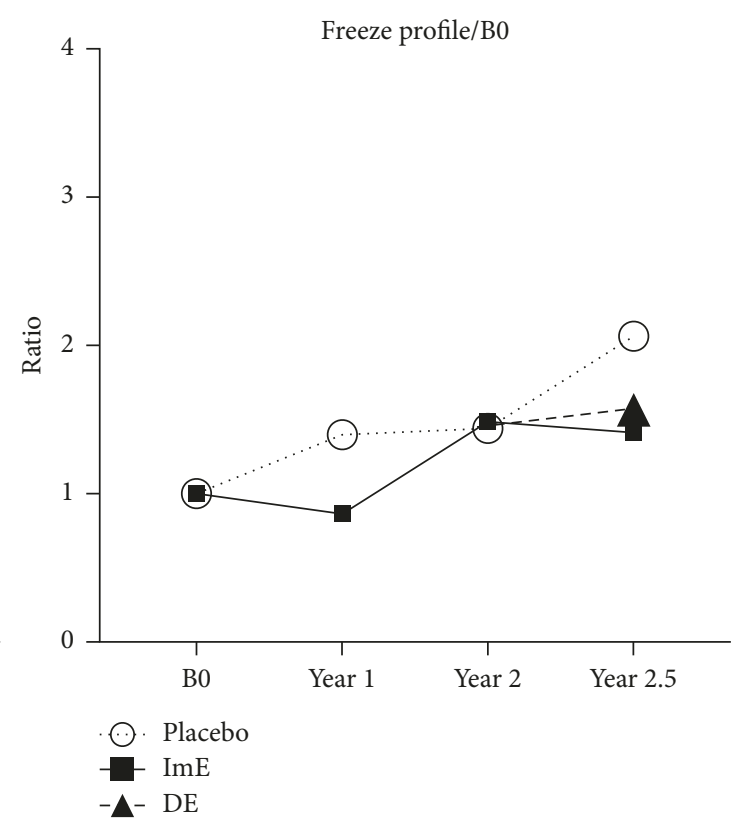

(b)

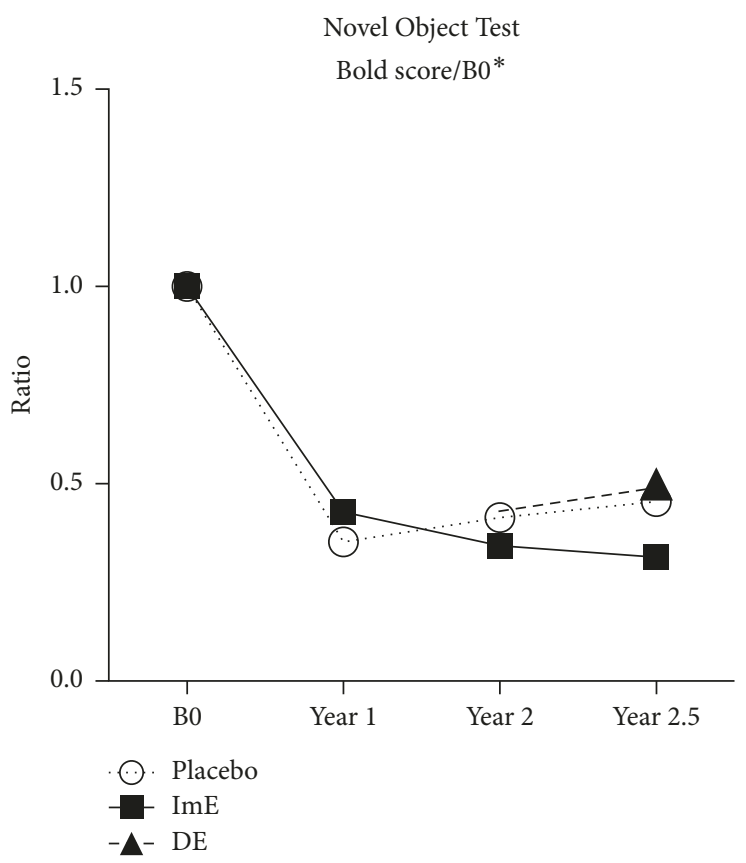

(c)

Figure 4: (a) Locomotion (normalized to B0) during the acclimation period was different across time $(p<0.041)$ and between treatment groups $(p<0.030)$. There was a significant difference between year 2 and year 2.5 (Tukey post hoc $p<0.035$ ). (b) There was no difference between treatments or across time in freezing after normalization to B0 due to a strange human profile presented. (c)The composite "Bold Score" normalized to B0 decreased significantly in all groups by the end of year 1 (time $p<0.0001$ ) and remained lower than B0 for the entire protocol. (Title* different by 2 -way ANOVA $(p<0.05)$; graph* different by post hoc test $(p<0.05)$ ).

indicates the length of time the animal remained completely motionless when exposed to the profile of the human intruder. There was no difference between treatment groups or across time with respect to this behavior.

At the bottom of Figure 4, panel C, the normalized "Bold Score" from the Novel Object Test is illustrated. "Bold Score" represents the individual's response to the objects. Higher scores correspond to increased propensity to inspect objects (and thus decreased anxiety toward the objects). The scores went from 0 (low boldness) to 12 (high boldness) There was a significant decrease in the normalized "Bold Score" in all animals after 1 year on WSD by 2 -way $\operatorname{ANOVA}(F(3,65)=$ 8.323; $p<0.0001)$. In other words, animals were more inhibited toward novelty after being on the WSD compared 


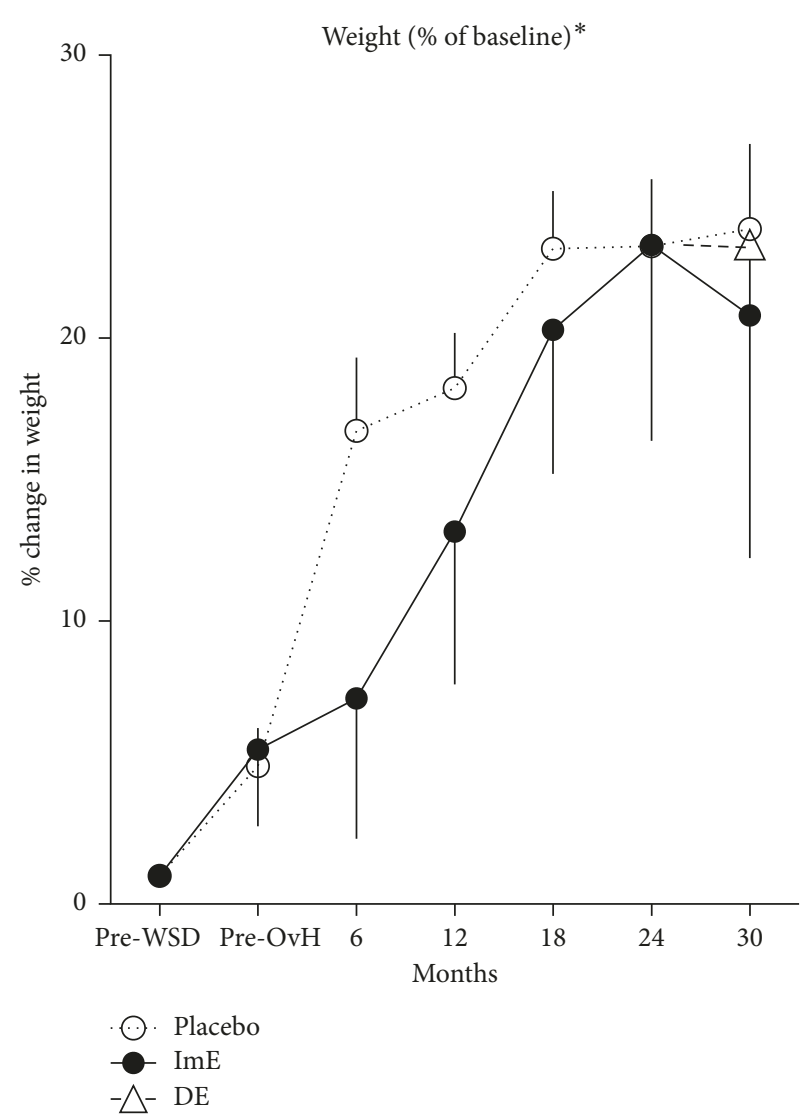

FIGURE 5: The average ( \pm SEM) body weights as percent of baseline. Body weights increased over time in both groups and reached a similar value at 30 months. However, $\mathrm{ImE}$ delayed the rate of increase until 12-18 months, at which time both groups exhibited similar weights. Administration of DE had no effect on body weight. There was a significant correlation between "consume" behavior and body weight. (Title* different by 2 -way ANOVA $(p<0.05)$.

with standard chow, but there were no differences between treatment groups.

The weights of the animals at 6-month intervals are illustrated in Figure 5, expressed as percent change from preWSD and analyzed with 2-way ANOVA. There was a significant effect of treatment $(p=0.030)$ and time $(p<0.0001)$, but no interaction. It appeared that $\mathrm{ImE}$ delayed the progression to obesity, although the final weights were the same. In addition, there was a significant correlation between "consume" behavior and weight $\left(p=0.003 ; r^{2}=0.64\right)$. However, there was no correlation between rank versus "alone" $\left(r^{2}=0.1338\right)$, "close social" $\left(r^{2}=0.1693\right)$, "locomote" $\left(r^{2}=0.1543\right)$, or "consume" $\left(r^{2}=0.0339\right)$. Also, there was no effect of treatment on the final dominance rank (chi-square $p=0.9$ ).

\section{Discussion}

This study examined the effects of diet and $\mathrm{E}$ treatment on home pen behavior and on behavior during anxiety tests in rhesus macaques. To create a naturalistic environment, we sought to socially house the animals in this study. Since the goal was to model menopause, older animals (at least 17 years) were chosen for OvH. A 17-year-old female monkey is approximately equal to a 51-year-old woman (1 monkey year $=3$ human years). After obtaining baseline measurements reflecting functions and behaviors when the animals were on normal monkey chow, the typical US diet, i.e. WSD, was initiated for all animals 6 weeks prior to OvH. E therapy was varied (e.g., either given immediately following $\mathrm{OvH}$ or after 2 years). Most of the animals were experimentally naïve and recruited from the outdoor breeding corrals. However, other animals were also used that were previously in cages, either alone or with a partner.

The greatest benefit to well-being is provided by complex social housing (groups) [28]. However, formation of the social groups was not straightforward. Several of the socialization attempts resulted in aggression, in which one or more animals had to be removed. There were factors that made these introduction attempts somewhat more challenging than most. Of the 28 animals finally assigned, 12 were used from recently reorganized outdoor breeding corrals, 12 had been in the outdoor corrals and brought inside to single housing for 1-3 months before the start of our study, and 4 had been single housed for at least 3 years. The latter 4 animals had been single-housed because they were not compatible with other female monkeys. Indeed, long periods of time housed without full contact with a conspecific can reduce future pair success in female rhesus macaques [24]. Ultimately, the 4 animals from prior single housing for 3 years were withdrawn from the study. Furthermore, all the monkeys had varying personalities, which plays a role in compatibility $[29,30]$. Some of the caged monkeys were somewhat "people-oriented," which made them ideal candidates for parts of this study (e.g., ability to work with investigators), but also made them challenging to socialize with other monkeys. While we thought that the extra space available in the pens (as opposed to cages) might help the socialization attempts, this was not true in all situations. Similar difficulties with group-housing captive rhesus macaques have been noted by several authors [29,31-33].

Another considerable factor was group stability. Group stability appeared affected by removal of an individual from the group for surgeries, which entailed recovery in a single cage for up to one week. This may confuse the remaining individuals (who could be unsure if their friend is coming back) with respect to rank. In the future, we will avoid removing only one animal at a time from the group pens for procedures that took hours or longer. Instead, all animals will be removed from the pen to single cages when one individual needs a procedure and the entire group will be returned to the pen together at the end of the day or next morning. We did not notice any stress responses from remaining animals when an individual was removed for a short period. Short protocols included temperament testing (i.e., Human Intruder and Novel Object), which is only 45 minutes in duration, venipuncture, physical exams, or getting weighed. Finally, in wild groups of rhesus macaques, males play an important part in maintaining social stability. We had no males in the group or even close to the pens to serve this function. Future studies will include an experienced, vasectomized male in each pen. 
A previous study with rhesus monkeys showed important differences in weight and metabolic parameters leading to type 2 diabetes (DBT2) depending on diet, housing, age, and sex [14]. In the same study, single-cage housing was strongly linked to emerging DBT2, and the authors attributed this to exercise. Also in cynomolgus macaques, single-cage housing increased stress and decreased exercise that in turn led to obesity, metabolic abnormalities, and a host of other stereotypic and self-injurious behaviors $[34,35]$. In the current study, the monkeys had room to exercise, but the WSD still led to changes in activity as described below.

With respect to surgical versus natural menopause in women, the major difference is the rate of decline of estradiol (E). With surgical menopause, the decline is abrupt, whereas with natural menopause, the decline is gradual and interspersed with random peaks of $\mathrm{E}$ until the ovary is completely depleted of follicles. Hormone therapy provided protection from cardiovascular events in ovary-intact perimenopausal women, but not in women who were surgically menopausal [36]. However, use of surgical menopause in this study was necessary because unlike humans, rhesus monkeys reach menopause so late in life that the risk of clinical attrition would increase greatly. This study was possible by using older monkeys and inducing menopause with surgery at the age of $\sim 50$ human years [37].

Key aspects of this study were the longitudinal aspect of the data collected and the ability of the animals to exercise if they desired. This enabled observation of changes over time due to WSD, as well as changes due to E therapy. Also, the ability to compare outcomes before and after WSD within a reasonable time frame was a significant improvement over clinical/human studies. An important change was observed in "consume" behavior. That is, the animals spent more time eating after switching to the WSD, and neither ImE nor DE therapy altered this behavior. These observations are consistent with another study that also reported that E had no effect on feeding behavior when the monkeys were fed a highly palatable diet [38].

Concomitantly, "locomote" behavior decreased, and there was a treatment effect. By 30 months, the placebo group tended to move about their pen less than the combined ImE + DE group. Hence, WSD per se promotes increased eating and decreased activity, with a modest effect of E on locomotion. A similar pattern was detected when the movement was monitored with activity collars, but it did not reach statistical significance at the 2-year time point [17]. These data suggest that a diet high in fat and processed sugar can promote overeating and a sedentary lifestyle, which may be exacerbated after menopause. Our results are also consistent with an earlier study in which administration of a selective estrogen receptor modulator (SERM) to ovariectomized monkeys caused weight loss, reduced adiposity, suppressed food intake, and increased activity [39]. It will be very important to understand the cellular and molecular aspects of this action. For example, chronic WSD and obesity increase cytokines, and E can block the cellular actions of cytokines [40-44].

Sociality was also affected by WSD and E therapy in our study. "Time alone" was relatively stable across time in the placebo-treated animals. However, it significantly increased over time in the ImE-treated animals. Inversely, "close social" behavior decreased in the ImE-treated animals, which corroborates the increase in "time alone." Seclusion and obesity/metabolic syndrome have been linked in a number of human studies. However, previous studies proposed that seclusion drives obesity, in both animal studies (single housing) [14] and in humans [45-48]. Our monkeys had access to social contact with at least one conspecific throughout the study, suggesting that the observed weight gain was largely due to WSD unlike earlier conclusions; but why did ImE increase "time alone?" Although somewhat counter-intuitive, spending time in close contact with conspecifics correlated with signs of depression in cynomolgus macaques [49]. Therefore, ImE could be antidepressive. Alternatively, it is possible that the observed increase in "time alone" has to do with their engagement in other activities, such as locomotion or vigilance (not to be confused with anxiety). Being in close social contact with another individual is incompatible with jumping about the pen (i.e., animals cannot engage in both behaviors simultaneously). Another potential reason for this finding is our method of observations (live for focal versus videotape for provoked tests). Had the observer, who was familiar to the monkeys, not been present, the monkeys may have behaved somewhat differently [50].

"Self-groom" was lower in the ImE group and decreased upon administration of DE to animals treated with placebo for 2 years. "Self-groom" is often considered a displacement behavior, indicative of anxiety similar to scratch and body shake [51] further suggesting that $\operatorname{ImE}$ decreased anxiety. Because "self-groom" was measured as a "duration," but other anxiety behaviors (e.g., scratch) were measured as "frequency," we were not able to combine these two behaviors into one larger anxiety category. Nonetheless, ImEtreated animals exhibited increased locomotion. One author postulated that pacing and other forms of stereotypy may be a coping mechanism for captive animals [52]. In the current study, there was no effect of time or treatment on stereotypic behavior. Taken together, these results suggest that ImE and DE helped the monkeys cope better with their environment by increasing activity.

Elevated anxiety in the placebo group was also indicated by behavior in the Human Intruder Test. That is, there was higher "locomotion" in the placebo-treated group during the period of acclimation to the test cage. This finding corroborates those of the focal observations and suggests that ImE might mitigate against anxiety. Excessive "freezing" while a potential threat (human intruder) is present, but has not yet noticed the subject, is also considered to be a sign of a fearful or anxious temperament [53]. However, there was no difference between treatment groups in "freezing" when exposed to the profile of a human intruder.

Therefore, both focal observations and HIT indicate that the placebo animals may have been more anxious. The lack of vigilance exhibited by the placebo group in the home pen could coexist with anxiety that manifests as self-grooming. Also of interest was the decrease in propensity to touch novel objects (e.g., decreased "Bold Score" on the novel object tests) after only one year, which was not different between 
treatment groups. Hence, it appears that WSD decreases boldness or exploratory behavior. Unfortunately, there was a lack of food motivation in attempts to train the monkeys in a Delayed Recall paradigm and in a Spatial Maze paradigm. Lack of food motivation may have also impacted their interest in the novel fruit.

Another aspect of boldness in humans is novelty seeking, which is inversely related to dopamine (DA) D2 receptors in the striatum. This inverse relationship is lost in addicts and obese subjects [54]. Drugs of abuse and palatable food with high-fat and high-sugar content activate DA reward circuitry [55]. However, in food addiction, we do not know the mechanism by which food activates the DA reward pathway [56]. We speculate that the decrease in boldness in our WSDfed macaques may reflect decreased DA D2 receptors in the ventral tegmental area (VTA) in a manner similar to the action of drugs of abuse, although the mechanism remains a mystery.

Rhesus macaques are a relatively aggressive species, and aggression toward conspecifics in the pens was highest as social ranks were resolved to the satisfaction of the group. Changes in aggression have been reported in cynomolgus macaques with diet and destabilized groupings [57]. Elevated dyad reciprocal aggression was also observed in unstable social groups [58], and food size can increase aggression [59]. However, after social groups had formed in this study (each pen contained animals with different treatments), there was no statistical difference in the frequency of initiated aggression between ImE-, DE-, or placebo-treated monkeys. Overall aggression decreased by $50 \%$ from $\mathrm{B} 0$ to the 2.5-year time point, but this difference was not significant between treatments. The largest delta of decrease in aggression initiations occurred in the placebo animals, which equaled 0.086 initiations $/ \mathrm{min}$. Although attrition by year 2.5 precluded statistical significance, it appeared that aggression was the inverse of close social contact. This relationship has been observed in subordinate cynomolgus macaques as well [60].

Altogether, WSD had serious effects on important behaviors in rhesus macaques such as socialization, consumption, exercise, grooming, and boldness that may parallel behaviors observed in humans eating a typical American diet. "Close social," "alone," "consume," and "locomote" were modified by ImE therapy, whereas boldness was not. ImEtreated WSD-fed animals exhibited an increase in the amount of "alone time" and in "locomote," as well as a decrease in "close social" and "self-groom." We attribute this constellation of behaviors to decreased anxiety relative to placebo-treated animals. In the provocation tests, the placebo group showed higher locomotion during the HIT acclimation period, a behavior that may further indicate anxiety in a different context. Clearly, much needed changes are needed in the American diet. In addition, the central neural and peripheral cellular and molecular mechanisms that lead to the results from eating a high fat-high sugar diet need resolution.

\section{Data Availability}

The data used to support the findings of this study are available from the corresponding author upon request.

\section{Additional Points}

(1) Old rhesus macaques were studied as a model of menopausal women who eat an obesogenic or "western-style diet" (WSD) and receive hormone therapy (HT) in the perimenopause or significantly later. (2) Focal and provoked behaviors were observed in a longitudinal manner over 2.5 years. (3) Animals received an empty Silastic capsule (placebo), an estradiol-filled capsule immediately upon ovohysterectomy (ImE), or an empty capsule for 2 years followed by an estradiol-filled capsule for 6 months (DE). (4) WSD increased consumption and decreased boldness, regardless of treatment. (5) Placebo-treated animals exhibited more sedentary and anxiety-type behaviors than ImEtreated animals. (6) DE-treated animals were similar to ImE animals in locomote and self-groom behaviors after 6 months, but not with respect to time alone or in close social.

\section{Conflicts of Interest}

The authors declare that they have no conflicts of interest.

\section{Acknowledgments}

The authors thank Kevin Mueller for his careful management of the colony and the veterinary staff of ONPRC for their clinical oversight of the resource animals. The authors also thank the Behavioral Services Unit for the help in maintaining the animals, as well as Madison DeCapo, Michelle Bermudez, Isabel Bernstein, Sabina Noll, and Jaclyn Shelton for their assistance in behavioral testing. Funding for this study was provided by the National Institutes of Health through grant R24 OD11895 to Cynthia L. Bethea and grant P51 OD11092 to the Oregon National Primate Research Center.

\section{References}

[1] S. M. Harman, E. A. Brinton, T. Clarkson et al., "Is the WHI relevant to HRT started in the perimenopause?," Endocrine, vol. 24, no. 3, pp. 195-202, 2004.

[2] S. M. Harman, F. Naftolin, E. A. Brinton, and D. R. Judelson, "Is the estrogen controversy over? Deconstructing the Women's Health Initiative study: a critical evaluation of the evidence," Annals of the New York Academy of Sciences, vol. 1052, no. 1, pp. 43-56, 2005.

[3] P. J. Schmidt and D. R. Rubinow, "Sex hormones and mood in the perimenopause," Annals of the New York Academy of Sciences, vol. 1179, no. 1, pp. 70-85, 2009.

[4] S. Shanmugan and C. N. Epperson, "Estrogen and the prefrontal cortex: towards a new understanding of estrogen's effects on executive functions in the menopause transition," Human Brain Mapping, vol. 35, no. 3, pp. 847-865, 2012.

[5] C. N. Epperson, Z. Amin, K. Ruparel, R. Gur, and J. Loughead, "Interactive effects of estrogen and serotonin on brain activation during working memory and affective processing in menopausal women," Psychoneuroendocrinology, vol. 37, no. 3, pp. 372-382, 2012.

[6] H. N. Hodis, W. J. Mack, V. W. Henderson et al., "Vascular effects of early versus late postmenopausal treatment with estradiol," New England Journal of Medicine, vol. 374, no. 13, pp. 1221-1231, 2016. 
[7] R. H. Foster and J. A. Balfour, "Estradiol and dydrogesterone. A review of their combined use as hormone replacement therapy in postmenopausal women," Drugs \& Aging, vol. 11, no. 4, pp. 309-332, 1997.

[8] I. Stipic, O. Polasek, M. Vulic, H. Punda, L. Grandic, and T. Strinic, "Estrogen replacement therapy improves pulmonary function in postmenopausal women with genital prolapse," Rejuvenation Research, vol. 15, no. 6, pp. 596-600, 2012.

[9] F. Engelmann, A. Rivera, B. Park, M. Messerle-Forbes, J. T. Jensen, and I. Messaoudi, "Impact of estrogen therapy on lymphocyte homeostasis and the response to seasonal influenza vaccine in post-menopausal women," PLoS One, vol. 11, no. 2, Article ID e0149045, 2016.

[10] M. S. Christianson, V. A. Mensah, and W. Shen, "Multiple sclerosis at menopause: potential neuroprotective effects of estrogen," Maturitas, vol. 80, no. 13, pp. 133-139, 2015.

[11] S. Smyth and A. Heron, "Diabetes and obesity: the twin epidemics," Nature Medicine, vol. 12, no. 1, pp. 75-80, 2006.

[12] M. J. Toth, A. Tchernof, C. K. Sites, and E. T. Poehlman, "Menopause-related changes in body fat distribution," Annals of the New York Academy of Sciences, vol. 904, no. 1, pp. 502-506, 2006.

[13] J. Jull, D. Stacey, and S. Beach, "Lifestyle interventions targeting body weight changes during the menopause transition: a systematic review," Journal of Obesity, vol. 2014, Article ID 824310, 16 pages, 2014.

[14] F. Yue, G. Zhang, J. E. Quintero, D. M. Gash, and Z. Zhang, "Role of social interaction, exercise, diet, and age on developing and untreated diabetes in cynomolgus monkeys," Experimental Gerontology, vol. 96, pp. 82-88, 2017.

[15] J. R. Kaplan, M. R. Adams, T. B. Clarkson, S. B. Manuck, and C. A. Shively, "Social behavior and gender in biomedical investigations using monkeys: studies in atherogenesis," Laboratory Animal Science, vol. 41, no. 4, pp. 334-343, 1991.

[16] C. L. Bethea, K. Mueller, A. P. Reddy, S. G. Kohama, and H. F. Urbanski, "Effects of obesogenic diet and estradiol on dorsal raphe gene expression in old female macaques," PLoS One, vol. 12, no. 6, Article ID e0178788, 2017.

[17] H. F. Urbanski, K. Mueller, and C. L. Bethea, "Effect of an obesogenic diet on circadian activity and serum hormones in old monkeys," Endocrine Connections, vol. 6, no. 6, pp. 380-383, 2017.

[18] J. Q. Purnell and C. L. Bethea, "Long-term western style diet (WSD) blocks the beneficial metabolic effects of immediate estradiol (E) replacement in older surgically menopausal macaques," in Proceedings of Annual Meeting of the Obesity Society, ASMBS, Los Angles, CA, USA, November 2015.

[19] J. Q. Purnell and C. L. Bethea, "Benefits of immediate versus delayed estradiol replacement on body composition and glucose metabolism in older surgically menopausal macaques on a long-term western style diet," in Proceedings of Annual Meeting of the Obesity Society, ASMBS, New Orleans, LA, USA, October 2016.

[20] J. Q. Purnell, H. F. Urbanski, and C. L. Bethea, "Effect of estrogen on body composition, activity, and hormone levels in non-human primates on a western-style diet," in Proceedings of Annual Meeting of the Obesity Society, ASMBS, Washington, DC, USA, October 2017.

[21] T. Yamada, M. Press, D. Vesselinovitch, and R. W. Wissler, "Quantitative ultrastructural analysis of coronary atherosclerotic involvement in two macaque species," Experimental and Molecular Pathology, vol. 48, no. 1, pp. 1-23, 1988.
[22] H. Uno and B. Poff, "Coronary arterial ectasia, a predominant type of coronary sclerosis in aged captive rhesus monkeys (Macaca mulatta)," American Journal of Pathology, vol. 111, no. 3, pp. 315-322, 1983.

[23] M. G. Bond, B. C. Bullock, D. A. Bellinger, and T. E. Hamm, "Myocardial infarction in a large colony of nonhuman primates with coronary artery atherosclerosis," American Journal of Pathology, vol. 101, no. 3, pp. 675-692, 1980.

[24] K. Coleman and P. J. Pierre, "Assessing anxiety in nonhuman primates,” ILAR Journal, vol. 55, no. 2, pp. 333-346, 2014.

[25] J. Altmann, "Observational study of behavior: sampling methods," Behaviour, vol. 49, no. 3, pp. 227-267, 1974.

[26] N. H. Kalin and S. E. Shelton, "Defensive behaviors in infant rhesus monkeys: environmental cues and neurochemical regulation," Science, vol. 243, no. 4899, pp. 1718-1721, 1989.

[27] K. Coleman, "Individual differences in temperament and behavioral management," in The Handbook of Primate Behavioral Management, S. J. Schapiro, Ed., pp. 95-114, CRC Press, Boca Raton, FL, USA, 2017.

[28] D. L. Hannibal, E. Bliss-Moreau, J. Vandeleest, B. McCowan, and J. Capitanio, "Laboratory rhesus macaque social housing and social changes: Implications for research," American Journal of Primatology, vol. 79, no. 1, article e22528, 2017.

[29] V. Reinhardt, "Group formation of previously single-caged adult rhesus macaques for the purpose of environmental enrichment," Journal of Experimental Animal Science, vol. 34, no. 3, pp. 110-115, 1991.

[30] M. A. Truelove, A. L. Martin, J. E. Perlman, J. S. Wood, and M. A. Bloomsmith, "Pair housing of macaques: a review of partner selection, introduction techniques, monitoring for compatibility, and methods for long-term maintenance of pairs," American Journal of Primatology, vol. 79, no. 1, article e22485, 2017.

[31] N. V. Meishvilli, V. G. Chalyan, and Y. Y. Rozhkova, "The causes of intragroup aggression in rhesus macaques," $\mathrm{Neu}$ roscience and Behavioral Physiology, vol. 39, no. 2, pp. 147151, 2009.

[32] Y. Choi, K.-H. Ahn, and J.-I. Lee, "Suppurative bite wound by repetitive aggression of dominance hierarchy during group housing in rhesus monkeys," Laboratory Animal Research, vol. 30, no. 4, pp. 181-184, 2014.

[33] L. A. Fairbanks, M. McGuire, and W. Kerber, "Effects of group size, composition, introduction technique and cage apparatus on aggression during group formations in rhesus monkeys," Psychological Reports, vol. 42, no. 1, pp. 327-333, 1978.

[34] C. M. Crockett, C. L. Bowers, M. Shimoji et al., "Behavioral responses of longtailed macaques to different cage sizes and common laboratory experiences," Journal of Comparative Psychology, vol. 109, no. 4, pp. 368-383, 1995.

[35] J. R. Kaplan and S. B. Manuck, "Status, stress, and atherosclerosis: the role of environment and individual behavior," Annals of the New York Academy of Sciences, vol. 896, no. 1, pp. 145-161, 1999.

[36] C. L. Shufelt, B. D. Johnson, and S. L. Berga, "Timing of hormone therapy, type of menopause, and coronary disease in women: data from the national heart, lung, and blood institute-sponsored women's ischemia syndrome Evaluation," Menopause, vol. 18, no. 9, pp. 943-950, 2011.

[37] W. A. Rocca, B. R. Grossardt, and L. T. Shuster, "Oophorectomy, menopause, estrogen, and cognitive aging: the timing hypothesis," Neurodegenerative Diseases, vol. 7, no. 1-3, pp. 163-166, 2010.

[38] Z. P. Johnson, J. Lowe, V. Michopoulos, C. J. Moore, M. E. Wilson, and D. Toufexis, "Oestradiol differentially 
influences feeding behaviour depending on diet composition in female rhesus monkeys," Journal of Neuroendocrinology, vol. 25, no. 8, pp. 729-741, 2013.

[39] E. L. Sullivan, J. Shearin, F. H. Koegler, and J. L. Cameron, "Selective estrogen receptor modulator promotes weight loss in ovariectomized female rhesus monkeys (Macaca mulatta) by decreasing food intake and increasing activity," American Journal of Physiology-Endocrinology and Metabolism, vol. 302, no. 7, pp. E759-E767, 2012.

[40] M. Debnath, S. Agrawal, A. Agrawal, and G. P. Dubey, "Metaflammatory responses during obesity: pathomechanism and treatment," Obesity Research and Clinical Practice, vol. 10, no. 2, pp. 103-113, 2016.

[41] R. Divella, R. De Luca, I. Abbate, E. Naglieri, and A. Daniele, "Obesity and cancer: the role of adipose tissue and adipocytokines-induced chronic inflammation," Journal of Cancer, vol. 7, no. 15, pp. 2346-2359, 2016.

[42] A. P. Miller, Y. F. Chen, D. Xing, W. Feng, and S. Oparil, "Hormone replacement therapy and inflammation: interactions in cardiovascular disease," Hypertension, vol. 42, no. 4, pp. 657-663, 2003.

[43] R. Nosalski and T. J. Guzik, "Perivascular adipose tissue inflammation in vascular disease," British Journal of Pharmacology, vol. 174, no. 20, pp. 3496-3513, 2017.

[44] P. Vural, C. Akgul, and M. Canbaz, "Effects of hormone replacement therapy on plasma pro-inflammatory and antiinflammatory cytokines and some bone turnover markers in postmenopausal women," Pharmacological Research, vol. 54, no. 4, pp. 298-302, 2006.

[45] M. Horsten, M. A. Mittleman, S. P. Wamala, K. SchenckGustafsson, and K. Orth-Gomer, "Social relations and the metabolic syndrome in middle-aged Swedish women," European Journal of Cardiovascular Risk, vol. 6, no. 6, pp. 391397, 1999.

[46] M. Pantell, D. Rehkopf, D. Jutte, S. L. Syme, J. Balmes, and N. Adler, "Social isolation: a predictor of mortality comparable to traditional clinical risk factors," American Journal of Public Health, vol. 103, no. 11, pp. 2056-2062, 2013.

[47] M. Sun, E. Y. Choi, D. J. Magee, C. W. Stets, M. J. During, and E. J. Lin, "Metabolic effects of social isolation in adult C57BL/6 mice," International Scholarly Research Notices, vol. 2014, Article ID 690950, 9 pages, 2014.

[48] S. Papadopoulos and L. Brennan, "Correlates of weight stigma in adults with overweight and obesity: a systematic literature review," Obesity, vol. 23, no. 9, pp. 1743-1760, 2015.

[49] C. A. Shively, T. C. Register, D. P. Friedman, T. M. Morgan, J. Thompson, and T. Lanier, "Social stress-associated depression in adult female cynomolgus monkeys (Macaca fascicularis)," Biological Psychology, vol. 69, no. 1, pp. 67-84, 2005.

[50] S. K. Iredale, C. H. Nevill, and C. K. Lutz, "The influence of observer presence on baboon (Papio spp.) and rhesus macaque (Macaca mulatta) behavior," Applied Animal Behaviour Science, vol. 122, no. 1, pp. 53-57, 2010.

[51] N. Kutsukake and D. L. Castles, "Reconciliation and variation in post-conflict stress in Japanese macaques (Macaca fuscata fuscata): testing the integrated hypothesis," Animal Cognition, vol. 4, no. 3-4, pp. 259-268, 2001.

[52] C. Poirier and M. Bateson, "Pacing stereotypies in laboratory rhesus macaques: implications for animal welfare and the validity of neuroscientific findings," Neuroscience and Biobehavioral Reviews, vol. 83, pp. 508-515, 2017.

[53] N. H. Kalin, S. E. Shelton, M. Rickman, and R. J. Davidson, "Individual differences in freezing and cortisol in infant and mother rhesus monkeys," Behavioral Neuroscience, vol. 112, no. 1, pp. 251-254, 1998.

[54] S. W. Savage, D. H. Zald, and R. L. Cowan, "Regulation of novelty seeking by midbrain dopamine D2/D3 signaling and ghrelin is altered in obesity," Obesity, vol. 22, no. 6, pp. 1452-1457, 2014.

[55] M. Michaelides, P. K. Thanos, N. D. Volkow, and G. J. Wang, "Translational neuroimaging in drug addiction and obesity," ILAR Journal, vol. 53, no. 1, pp. 59-68, 2012.

[56] J. H. Baik, "Dopamine signaling in food addiction: role of dopamine D2 receptors," BMB Reports, vol. 46, no. 11, pp. 519-526, 2013.

[57] J. R. Kaplan, S. B. Manuck, and C. Shively, "The effects of fat and cholesterol on social behavior in monkeys," Psychosomatic Medicine, vol. 53, no. 6, pp. 634-642, 1991.

[58] B. A. Beisner, J. Jin, H. Fushing, and B. McCowan, "Detection of social group instability among captive rhesus macaques using joint network modeling," Current Zoology, vol. 61, no. 1, pp. 70-84, 2015.

[59] J. W. Mathy and L. A. Isbell, "The relative importance of size of food and interfood distance in eliciting aggression in captive rhesus macaques (Macaca mulatta)," Folia Primatologica, vol. 72, no. 5, pp. 268-277, 2001.

[60] C. A. Shively, "Social subordination stress, behavior and central monoaminergic function in female cynomolgus monkeys," Biological Psychiatry, vol. 44, no. 9, pp. 882-891, 1998. 


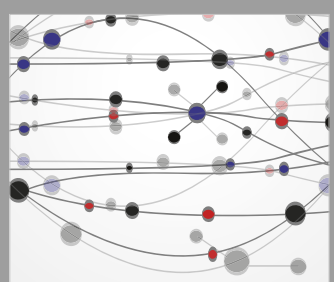

The Scientific World Journal
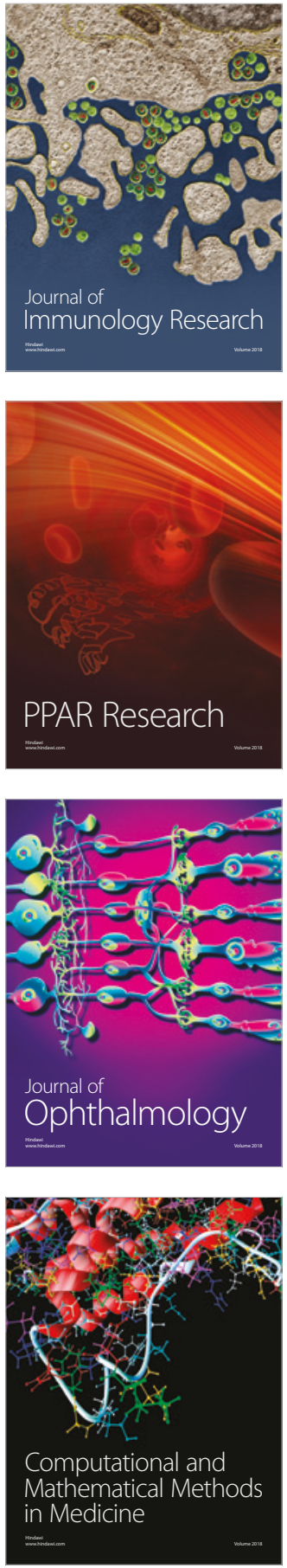

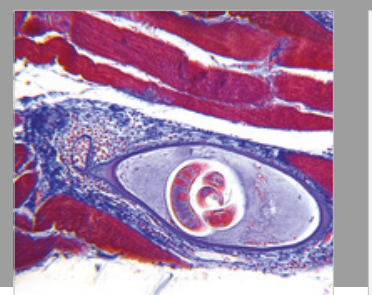

Gastroenterology Research and Practice

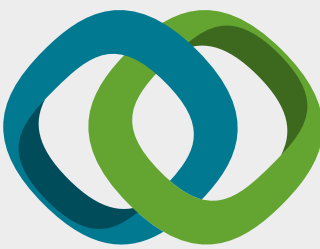

\section{Hindawi}

Submit your manuscripts at

www.hindawi.com
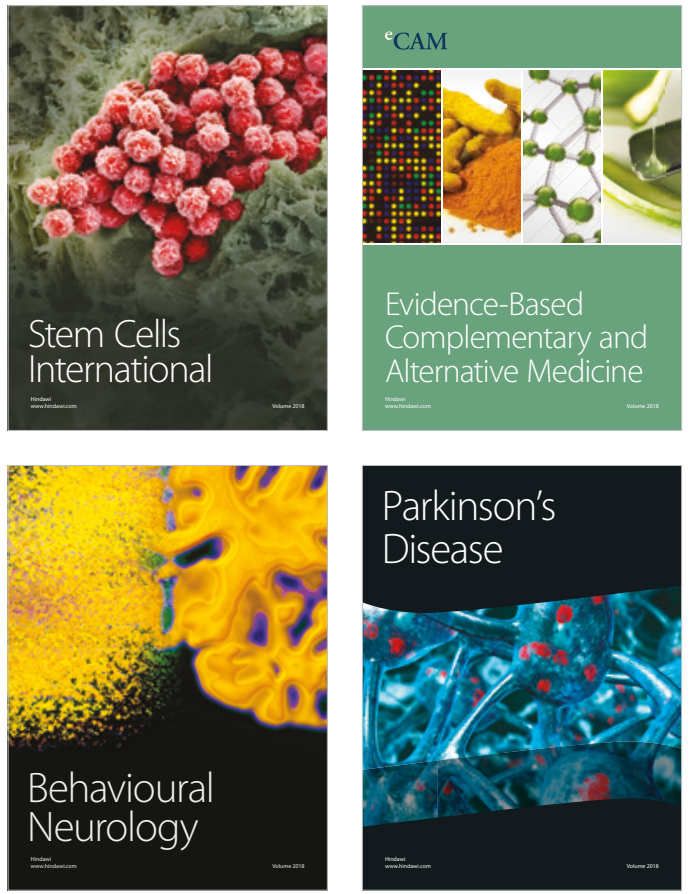

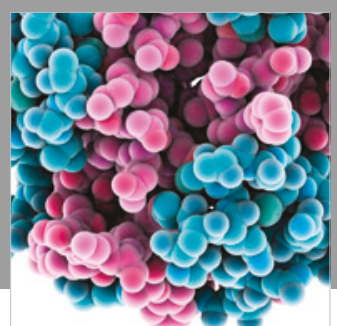

ournal of

Diabetes Research

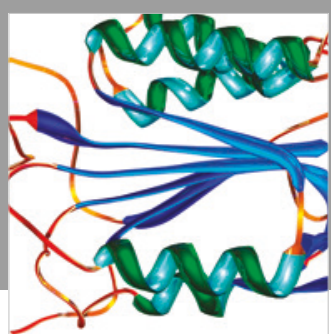

Disease Markers
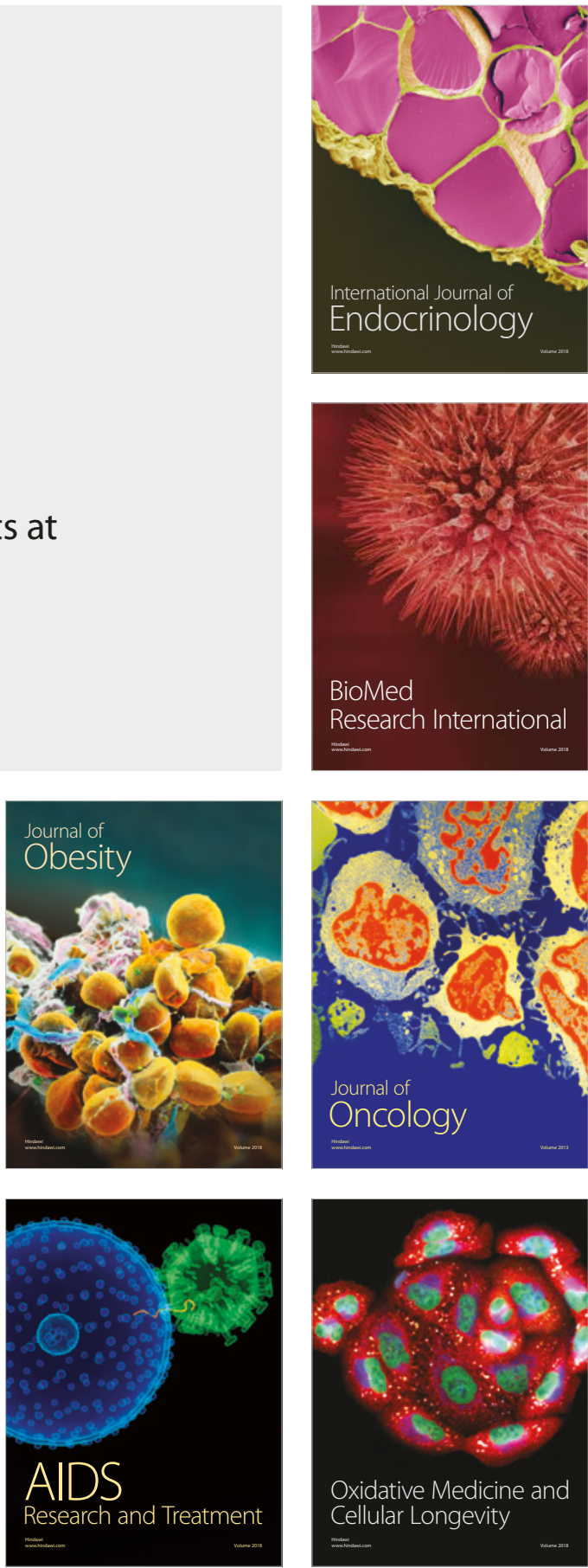